\title{
CHROMOSPHERIC HEATING IN LATE-TYPE STARS: \\ EVIDENCE FOR MAGNETIC AND NONMAGNETIC \\ SURFACE STRUCTURE
}

\author{
MANFRED CUNTZ \\ Joint Institute for Laboratory Astrophysics \\ University of Colorado and NIST \\ Boulder, CO 80309-0440, USA
}

\begin{abstract}
The aim of this paper is to evaluate recent observational and theoretical results concerning the physics of chromospheric heating as inferred from IUE, HST-GHRS and ROSAT data. These results are discussed in conjunction with theoretical model calculations based on acoustic and magnetic heating to infer some conclusions about the magnetic and nonmagnetic surface structure of cool luminous stars. I find that most types of stars may exhibit both magnetic and nonmagnetic structures. Candidates for pure nonmagnetic surface structure include $M$-type giants and supergiants. M-type supergiants are also ideal candidates for identifying direct links between the appearance of hot spots on the stellar surface (perhaps caused by large convective bubbles) and temporarily increased chromospheric heating and emission.
\end{abstract}

\section{Introduction}

An important outstanding problem in stellar astrophysics concerns the identification of processes responsible for heating stellar chromospheres and coronae and for driving stellar winds. Major advances during the past $20 \mathrm{yr}$ have been made, in part due to different types of observations as obtained by IUE, ROSAT, HST-GHRS, and in part due to sophisticated theoretical modelling. Based on these results, it has been concluded that two different types of processes exist: magnetic processes and nonmagnetic processes. In nonpulsating stars, the nonmagnetic processes largely coincide with acous- 
tic heating. It is now strongly believed that these processes have different importance in stars of different effective temperature, gravity, and rotation rate and change dramatically during the course of stellar evolution. On the other hand, magnetic and nonmagnetic processes are manifestations of distinct stellar surface structures, which are key in determining the amount of mechanical energy and momentum input to the atmospheres and winds.

The basic goal of this paper is to summarize key results for late-type stars. I will give four propositions, which are discussed in detail. In particular, I will consider observational and theoretical results obtained within the last two years.

\section{Four propositions}

The four propositions are:

1. The basal chromospheric emission in main-sequence stars can probably be attributed to pure acoustic heating. Nonetheless, these stars should also possess magnetic-field related surface structure, which is probably required to heat the corona.

2. When solar-type stars evolve up the giant branch, their magnetic fields decay and the generation of acoustic energy increases drastically. As a consequence, the heating in single late-type giants and supergiants above the coronal dividing line is dominated increasingly by nonmagnetic processes.

3. Most types of stars located above the coronal dividing line should nevertheless have a small magnetic field coverage. In case of noncoronal $\mathrm{K}$ giants with basal chromospheric flux emission, the presence of magnetic fields can be inferred from the magnitude of chromospheric turbulence and the presence of mass loss.

4. Candidates for pure nonmagnetic surface structure include M-type giants and supergiants. $M$ supergiant stars are also ideal candidates for identifying direct links between the appearance of hot spots on the stellar surface (perhaps caused by large convective bubbles) and temporarily increased chromospheric heating and emission.

\section{Evaluation of the propositions}

\subsection{PROPOSITION 1}

It is generally presumed that the outer atmospheres of main-sequence stars are heated both by magnetic and nonmagnetic processes. In case of the Sun, Rammacher \& Ulmschneider (1992) and Carlsson \& Stein $(1992,1995)$ have shown that the $\mathrm{Mg}$ II and Ca II line emission above the intranetwork regions 
can be reproduced successfully by acoustic energy dissipation. Additional results are given by Rutten (1996, this volume). Magnetic regions can be heated by magnetic flux-tube waves and other magnetic-field related mechanisms (see, e.g., Narain \& Ulmschneider 1990).

For main-sequence stars other than the Sun, important observational results which point to both magnetic and nonmagnetic heating have been summarized by Schrijver (1987) and Rutten et al. (1991). They presented a detailed statistical analysis of the flux-flux and flux-color relations derived from selected emission lines for a large sample of late-type stars. They found, particularly from their Mg II and Ca II emission line studies, that the statistical correlations can be understood best when the stellar emission line fluxes are assumed to consist of two components: a "basal" flux, which is independent of stellar rotation, and a "magnetic" flux, which depends upon rotation and age. The basal flux could be identified unequivocally as an intrinsic property of the stars and is not an artifact of detection limits. Schrijver (1987) and Rutten et al. (1991) have argued that the basal flux emission might be attributable to pure acoustic heating.

This proposal has found support by detailed hydrodynamic studies. Buchholz \& Ulmschneider (1994) and Buchholz (1995) have calculated acoustic heating models for stars of spectral type F5 V, G0 V, G5 V, $\mathrm{K} 0 \mathrm{~V}, \mathrm{~K} 5 \mathrm{~V}$, and M0 V. By adopting representative timesteps of the wave solution, they calculated synthetic Mg II $k$ and Ca II K line profiles and $\mathrm{Mg}$ II and Ca II emission line fluxes. These line fluxes are then compared with the empirical basal flux line for $\mathrm{Mg}$ II and $\mathrm{Ca}$ II given by Rutten et al. (1991). Buchholz \& Ulmschneider found that the agreement between the computed $\mathrm{Mg}$ II and $\mathrm{Ca}$ II fluxes and the empirical $\mathrm{Mg}$ II and $\mathrm{Ca}$ II basal flux line is remarkably good for all stars considered. This result is remarkable as the stars considered have spectral types ranging from F5 V to M0 V implying enormous differences in their atmospheric structure. For inactive M dwarf stars, the study by Mullan \& Cheng (1993) also attributes chromospheric radiative energy losses to acoustic heating. They found that their theoretical models are capable of reproducing the observed Mg II Ly- $\alpha$ flux-flux relationship.

On the other hand, there is evidence that also chromospheric basal flux stars have both magnetic and nonmagnetic surface structures because these stars show coronal X-ray emission, which is difficult to reconcile with purely nonmagnetic heating. Rutten et al. (1991), who identified basal flux components in the lines of $\mathrm{Mg}$ II, Ca II, C II, Si II, C IV, and Si IV, found no evidence that a basal flux component also exists in soft X-ray emission. This result is supported by the deduced flux-flux relationships and is consistent with results from other studies. Stępień \& Ulmschneider (1989) calculated the expected X-ray emission from acoustic wave models for F, G, and $\mathrm{K}$ 
main-sequence stars and a limited number of giants by assuming shortperiod acoustic shock waves. They found that the calculated and observed X-ray fluxes differ by two orders of magnitude. Hammer \& Ulmschneider (1991) also assessed the possibility of acoustically heated coronae. They found that for solar-type stars, the resulting coronal base pressures would to be extremely low, which would make these acoustically heated coronae unobservable. Other arguments against acoustic heating of stellar coronae rely on the extremely complex coronal topology inferred from solar observations, which can directly be linked to magnetic structures and can barely be reproduced by pure nonmagnetic heating.

On the other hand, evidence is now available that acoustic heating might indeed contribute (or even dominate?) the coronal emission in stars with an extremely low level of X-ray activity. Mullan \& Cheng (1994a,b) have studied models for extremely inactive M-type main-sequence stars and models appropriate to Procyon (F5 IV-V). The heating model for Procyon is found to explain various spectral features of this star, including the soft $\mathrm{X}$-ray emission and the observed fluxes in Mg II and $\mathrm{H}$ Ly- $\alpha$. They also found that the wave period is an extremely sensitive input parameter and its value controls whether the observed and computed X-ray fluxes are in agreement or not. These results clearly contradict other findings which show the impossibility of acoustic heating models to explain the observed X-ray emission. Further studies are needed to resolve this issue.

\subsection{PROPOSITION 2}

When solar-type stars evolve up the giant branch, their angular momentum decreases, in part due to the increase in the moment of inertia and to magnetic braking resulting from the onset of massive stellar winds (Schrijver 1993, MacGregor \& Charbonneau 1994, Charbonneau et al. 1996). Charbonneau et al. focussed on stars with $M / M_{\odot}=0.8$ and 2.0 , which evolve to and apart from the main-sequence. Significant changes in the moment of inertia are found to occur as a star evolves leading to strong spin-up on the pre-main sequence and strong spin-down on the subgiant and giant branches.

In recent calculations for luminosity class (LC) IV stars, it is found that stars with $M / M_{\odot} \lesssim 1.2$ remain redward of the rotational dividing line (which separates stars with and without high rotational velocities as expressed by $v \cdot \sin i$ ) through their post-main sequence evolution up to the giant branch. Therefore, ample time is available for these low mass stars, while on the main-sequence, to loose angular momentum. More massive stars $\left(1.2 \lesssim M / M_{\odot} \lesssim 2.0\right)$ spend their main-sequence lifetime blueward of the rotational dividing line. For these stars, the increase in the moment of 
inertia is too small to account for the observed drop in the rotation rates (Rutten \& Pylyser 1988). This result points to angular momentum loss due to the onset of massive stellar winds as these stars evolve toward the red giant branch (Gray \& Nagar 1985; see also Charbonneau et al. 1996). For more massive stars, similar results have been found by Gray (1989), Pasquini et al. (1990) and Schrijver \& Pols (1993).

Schrijver (1993) summarized key aspects of the stellar angular momentum evolution and discussed relationships to observed properties. He found that the loss of angular momentum in single stars (or stars showing no or only marginal interaction with other components) evolving up the red giant branch comfortably explains various observational results including the decrease in the surface equatorial rotational velocity (Schrijver \& Pols 1993) and the decrease in Ca II emission (Rutten 1987, Strassmeier et al. 1994). Stars evolving up the giant branch eventually cross the coronal dividing line (CDL), first indicated by Linsky \& Haisch (1979). Stars above the CDL barely show X-ray emission (Maggio et al. 1990, Ayres et al. 1991, $1995)$ and in most cases provide no evidence for transition layer emission (Haisch et al. 1990, Ayres et al. 1995). These results clearly indicate a drastic decline in the surface magnetic field strength due to the stellar evolution. Rosner et al. $(1991,1995)$, furthermore, argued in favor of changes in magnetic field topology in stars crossing the CDL, which are found to be relevant to the outer atmospheric dynamics. An interesting result has also been found by Hatzes \& Cochran (1993), who presented a tentative determination of rotational velocities in selected $\mathrm{K}$ giants based on the observed rotational modulation of hot surface features. They found rotation periods of $223 \mathrm{~d}$ for $\alpha$ Boo (K1.5 III), $643 \mathrm{~d}$ for $\alpha$ Tau (K5 III), and $558 \mathrm{~d}$ for $\beta$ Gem (K0 III) compared to $\simeq 25 \mathrm{~d}$ for the Sun (G2 V). This result strengthens the case for the loss of angular momentum in evolving late-type stars.

In addition to the decrease in magnetic field-related activity, another effect occurs when stars evolve up the giant branch: this is the increased production of acoustic energy due to higher convective velocities caused by reduced surface gravity. Renzini et al. (1977) found that the photospheric acoustic energy flux scales as $g_{*}^{-0.7}$ or $g_{*}^{-1.2}$, depending on whether $\mathrm{H}_{2}$ molecules are present or not. Bohn (1984) deduced a gravity dependence of the acoustic energy flux of $g_{*}^{-0.5}$, whereas an improved study by Ulmschneider et al. (1996) led to a gravity dependency between $g_{*}^{-0.5}$ and $g_{*}^{-1.5}$, depending on stellar structure model.

Judge \& Stencel (1991) have presented a detailed statistical analysis of the global thermodynamic properties of the outer atmospheres and winds of late-type giant and supergiant stars with spectral types $\mathrm{K}, \mathrm{M}$ and $\mathrm{C}$, considering empirical results for the stellar mass loss rates, the chromospheric radiative energy losses and the terminal flow speed of the winds. 
Concerning the chromospheric $\mathrm{Mg}$ II fluxes, they found a rather smooth decline with the effective temperature and, most importantly, a smooth joining with the Mg II basal flux limit given by Schrijver (1987), a suspected indicator of pure acoustic heating (see above). This result points again to the importance of acoustic energy dissipation in stars that have evolved along the red giant branch.

\subsection{PROPOSITION 3}

While acoustic energy dissipation can explain the chromospheric emission losses in basal flux stars, there is evidence that even chromospheric basal flux stars such as noncoronal $\mathrm{K}$ giants might have a low level of magnetic activity remaining, as various observed features cannot be explained otherwise. The evidence includes the presence of mass loss in these stars and the high level of atmospheric turbulence. Cuntz (1990) has shown that the mass loss rates in inactive $\mathrm{K}$ giants as inferred from acoustic heating models are many orders of magnitude below the values derived from observations because most of the acoustic energy is dissipated immediately beyond the photospheres. This energy is therefore unavailable to overcome the gravitational potential of the star. Preliminary estimates of this behavior have already been given by Hartmann \& MacGregor (1980). Sutmann \& Cuntz (1995) have meanwhile rediscussed this issue in the case of $\alpha$ Boo (K1.5 III). Besides the dissipation of short-period acoustic waves, they also considered the role of long-period photospheric oscillation modes, which have been observed by Belmonte et al. (1990) and others. These modes have very large periods, i.e. close to or larger than the acoustic cut-off period, but extremely small amplitudes $\left(<100 \mathrm{~m} \mathrm{~s}^{-1}\right)$. As a consequence, the modes are trapped and are unimportant for the atmospheric dynamics.

It has also been found that acoustic heating models appear to be unable to explain the magnitude of observed chromospheric turbulence. Recent GHRS data for $\alpha$ Tau (K5 III) revealed a high level of chromospheric turbulence (i.e., $\simeq 24 \mathrm{~km} \mathrm{~s}^{-1}$ ) (Carpenter et al. 1991), which appears to be inconsistent with acoustic wave calculations of Judge \& Cuntz (1993) who calculated 1-D time-dependent wave models based on acoustic shock wave dissipation. They calculated synthetic C II] profiles near $2325 \AA$ and compared the results with the observations. Judge \& Cuntz found that the turbulent velocities inferred from the theoretical models are $\simeq 5 \mathrm{~km} \mathrm{~s}^{-1}$ or somewhat less, depending on the model parameters, which is a factor of 5 smaller than observed. In addition, the centroid of the computed emission profiles were found to be blueshifted by $\sim 1-2 \mathrm{~km} \mathrm{~s}^{-1}$, whereas the observed profiles are redshifted by $3.9 \pm 0.4 \mathrm{~km} \mathrm{~s}^{-1}$. They concluded that one (or more) of the model assumptions must be incorrect, suggesting that 
either (i) the chromospheric dynamics are dominated by 3-D or horizontal flow patterns not represented by the models, or (ii) that the magnetic field plays an important role in the chromospheric dynamics. On the other hand, the models of Judge \& Cuntz ignored important thermodynamic effects in the atmosphere, including noninstantaneous ionization of hydrogen behind shocks, which may affect their conclusions. Studies are now underway to overcome this technical restriction.

\subsection{PROPOSITION 4}

For stars with extremely low gravity, one must describe the generation of mechanical energy by stellar surface structure in more detail. First, we assume that most, if not all M-type giants and supergiants possess a very low level of magnetic-field related activity as a consequence of the decrease of angular momentum during evolution along the first giant branch (see Proposition 2). Nonetheless, some small magnetic field coverage may still exist in these stars, which is potentially relevant for the outer atmospheric dynamics as discussed in the framework of Alfvén wave driven wind models (Hartmann \& Avrett 1984, Charbonneau \& MacGregor 1995). Reliable magnetic field measurements do, however, not yet exist for these stars (Marcy \& Bruning 1984, among other literature).

Another important question concerning these stars is the structure of stellar convection. Based on observational photospheric data which show significant variabilities, Schwarzschild (1975) suggested that the size scale of the convective elements in the photospheres of red giants and supergiants might be extremely large that only a modest number of cells can be present at any one time on the stellar surface. This result is in sharp contrast to the case of the Sun (and of similar stars), where the number of granules simultaneously present on the surface exceeds two million. Subsequent support for Schwarzschild's suggestion was given by Antia et al. (1984) based on time-independent stellar convection zone calculations.

Schwarzschild's conjecture has meanwhile gained significant observational support: Buscher et al. (1990) and Wilson et al. (1992) presented results from a high-resolution imaging monitoring program for $\alpha$ Ori (M2 Iab) using the Nasmyth focus on the William Herschel Telescope (WHT), which shows the time evolution of a bright surface feature over a period of nearly two years (i.e. from February 1989 to January 1991). The feature was identified at four different wavelengths between 546 and $710 \mathrm{~nm}$ and was found to cover at least $10 \%$ of the stellar disk. A very significant result obtained by Toussaint \& Reimers (1989) is that the appearance of the hot spot on $\alpha$ Ori given by Buscher et al. (1990) coincides with enhanced Ca II K emission. Toussaint \& Reimers observed a substantial increase in 


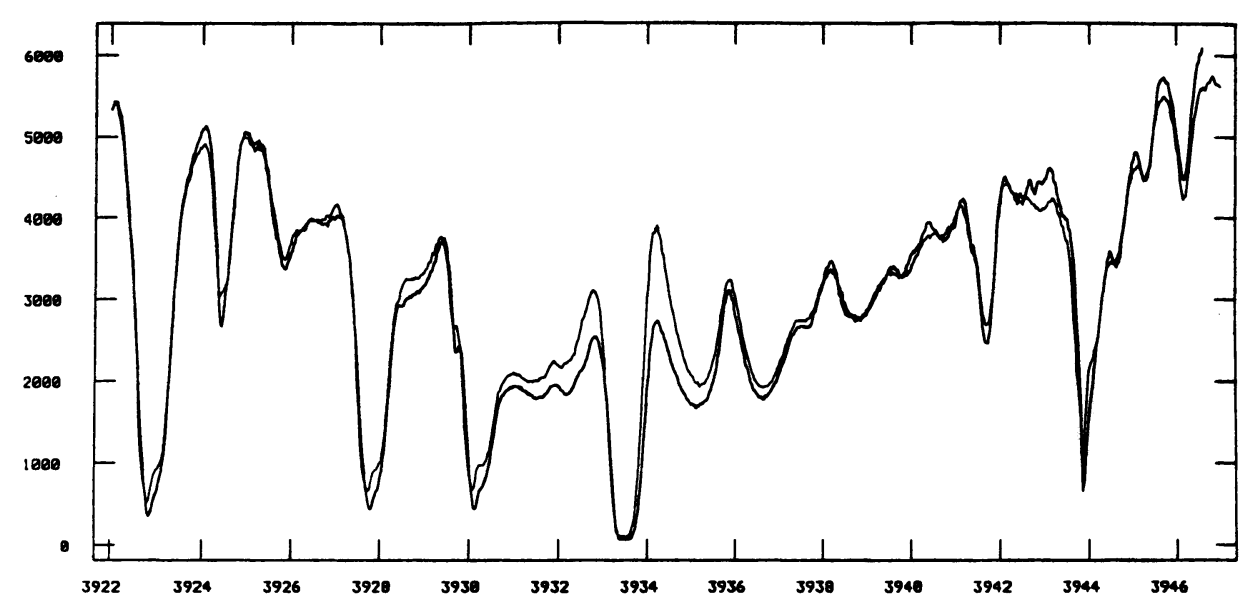

Figure 1. Ca II flux in $\alpha$ Ori (M2 Iab) on February 1989 (strong solid line) and February 1988 (weak solid line) given by Toussaint \& Reimers (1989). This event is possibly caused by increased acoustic energy production due to a hot surface feature

the chromospheric Ca II K2 emission feature between February 1988 and February 1989 corresponding to nearly $35 \%$ of the total Ca II K flux (Schrijver 1995) (see Fig. 1). Note that both dates refer to a similar phase in the $\alpha$ Ori pulsation cycle, which reduces the possibility that the increased Ca II emission is caused by photospheric pulsation considerably.

I believe that the following important implications can be inferred from the previous discussion. First of all, one must accept the idea that a timeaveraged, i.e. globally constant, acoustic energy flux is a meaningless concept in stars which are most evolved. In reality, large convective bubbles suddenly appear at stellar surface, which lead to the generation of significant nonradiative energy on localized horizontal scales. These events then produce localized episodic chromospheric heating. In this case, one can make a direct link between atmospheric energy dissipation and the appearance of convective stellar surface structures.

These results have additional relevance considering observations presented during the Vienna meeting: Wilson et al. (1996) presented results from a subsequent study, providing evidence for three spots on the surface of $\alpha$ Ori. Another study of $\alpha$ Ori presented by Klückers et al. (1995) uses Speckle imaging observations at the WHT. These results also provide evidence for an $\alpha$ Ori surface feature occurring on the nights of January 19 and 20, 1995. Further results were given by Gilliland \& Dupree (1996, this volume). He presented significant evidence for a spot 
on $\alpha$ Ori using the Faint Object Camera on HST to obtain images at 2530 $\AA$ (continuum) and $2780 \AA$ (Mg II emission). These and subsequent results should lead to a better understanding of relationships better stellar surface structure and chromospheric heating and activity.

\section{Conclusions}

It has been the aim of this paper to discuss critically the presence of magnetic and nonmagnetic surface structure in late-type stars by evaluating results on chromospheric heating. It is likely that most types of stars have both magnetic and nonmagnetic regions present which are important for explaining the strength of the chromospheric emission, the atmospheric turbulence and the stellar mass loss behavior. Measurements of the surface magnetic field strength are still difficult, if not impossible, for these stars as discussed by Marcy \& Bruning (1984), among others. Candidates for pure nonmagnetic surface structure include M-type giants and supergiants. $M$ supergiant stars are also ideal candidates for identifying direct links between the appearance of hot spots on the stellar surface (perhaps caused by large convective bubbles) and temporarily increased chromospheric heating and emission.

Acknowledgements: Support for this work was provided by NASA through grant number AR-5285.02-93A from the Space Telescope Science Institute, which is operated by the Association of Universities for Research in Astronomy, Inc., under NASA contract NAS5-26555. Additional support was provided by the NASA Astrophysical Theory Program to the University of Alabama in Huntsville.

\section{References}

Antia, H.M., Chitre, S.M., \& Narasimha, D. 1984, ApJ, 282, 574

Ayres, T.R., Fleming, T.A., \& Schmitt, J.H.M.M. 1991, ApJ, 376, L45

Ayres, T.R., et al. 1995, ApJS, 96, 223

Belmonte, J.A., Jones, A.R., Pallé, P.L., \& Cortés, T.R. 1990, ApJ, 358, 595

Bohn, H. U. 1984, A\&A, 136, 338

Buchholz, B. 1995, Ph.D. thesis, Univ. Heidelberg

Buchholz, B., \& Ulmschneider, P. 1994, Cool Stars, Stellar Systems, and the Sun VIII, ed. J.-P. Caillault, ASPCS 64, p. 363

Buscher, D.F., Haniff, C.A., Baldwin, J.E., \& Warner, P.J. 1990, MNRAS, 245, 7P

Carlsson, M., \& Stein, R.F. 1992, ApJ, 397, L59

Carlsson, M., \& Stein, R.F. 1995, ApJ, 440, L29

Carpenter, K.G., et al. 1991, ApJ, 377, L45

Charbonneau, P., \& MacGregor, K.B. 1995, ApJ, in press

Charbonneau, P., Schrijver, C.J., \& MacGregor, K.B. 1996, in Cosmic Winds and the Heliosphere, ed. J.R. Jokipii et al. (Tucson: The University of Arizona Press), in press Cuntz, M. 1990, ApJ, 353, 255 
Gilliland, R.L., \& Dupree, A.K. 1996, this volume

Gray, D.F. 1989, ApJ, 347, 1021

Gray, D.F., \& Nagar, P. 1985, ApJ, 298, 756

Haisch, B.M., et al. 1990, ApJ, 361, 570

Hammer, R., \& Ulmschneider, P. 1991, Mechanisms of Chromospheric and Coronal Heating, ed. P. Ulmschneider et al. (Springer: Berlin), p. 344

Hartmann, L., \& Avrett, E.H. 1984, ApJ, 284, 238

Hartmann, L., \& Mac Gregor, K.B. 1980, ApJ, 242, 260

Hatzes, A.P., \& Cochran, W.D. 1993, ApJ, 413, 339

Judge, P.G., \& Cuntz, M. 1993, ApJ, 409, 776

Judge, P.G., \& Stencel, R.E. 1991, ApJ, 371, 357

Klückers, V.A., Edmunds, M.G., Wooder, N.J., \& Morris R. 1995, Stellar Surface Structure, Poster Proceedings, ed. K.G. Strassmeier, p. 48

Linsky, J.L., \& Haisch, B.M. 1979, ApJ, 229, L27

MacGregor, K.B., \& Charbonneau P. 1994, Cool Stars, Stellar Systems, and the Sun VIII, ed. J.-P. Caillault, ASPCS 64, p. 174

Maggio, A., et al. 1990, ApJ, 348, 253

Marcy, G.W., \& Bruning, D.H. 1984, ApJ, 281, 286

Mullan, D.J., \& Cheng, Q.Q. 1993, ApJ, 412, 312

Mullan, D.J., \& Cheng, Q.Q. 1994a, ApJ, 420, 392

Mullan, D.J., \& Cheng, Q.Q. 1994b, ApJ, 435, 435

Narain, U., \& Ulmschneider, P. 1990, Space Sci. Rev., 54, 377

Pasquini, L., Brocato, E., \& Pallavicini, R. 1990, A\&A, 234, 277

Rammacher, W., \& Ulmschneider, P. 1992, A\&A, 253, 586

Renzini, A., Cacciari, C., Ulmschneider, P., \& Schmitz, F. 1977, A\&A, 61, 39

Rosner, R., An, C.-H., Musielak, Z.E., Moore, R.L., \& Suess, S.T. 1991, ApJ, 372, L91

Rosner, R., Musielak, Z.E., Cattaneo, F., Moore, R.L., \& Suess, S.T. 1995, ApJ, 442, L25

Rutten, R.G.M. 1987, A\&A, 177, 131

Rutten, R.G.M., \& Pylyser, E. 1988, A\&A, 191, 227

Rutten, R.G.M., Schrijver, C.J., Lemmens, A.F.P., \& Zwaan, C. 1991, A\&A, 252, 203

Rutten, R.J. 1996, this volume

Schrijver, C.J. 1987, A\&A, 172, 111

Schrijver, C.J. 1993, Inside the Stars, ed. W.W. Weiss \& A. Baglin, ASPCS 40, p. 328

Schrijver, C.J. 1995, A\&AR, 6, 181

Schrijver, C.J., \& Pols, O.R. 1993, A\&A, 278, 51

Schwarzschild, M. 1975, ApJ, 195, 137

Steppień, K., \& Ulmschneider, P. 1989, A\&A, 216, 139

Strassmeier, K.G., Handler, G., Paunzen, E., \& Rauth, M. 1994, A\&A, 281, 855

Sutmann, G., \& Cuntz, M. 1995, ApJ, 442, L61

Toussaint, F., \& Reimers, D. 1989, A\&A, 226, L17

Ulmschneider, P., Theurer, J., \& Musielak, Z.E. 1996, A\&A, submitted

Wilson, R.W., Baldwin, J.E., Buscher D.F., \& Warner, P.J. 1992, MNRAS, 257, 369

Wilson, R.W., Dhillon, V.S., Haniff, C.A., \& Baldwin, J.E. 1996, in preparation 